\title{
ROL DEL COLGAJO PERFORANTE PEDICULADO EN HÉLICE (PROPELLER FLAP) EN RECONSTRUCCIÓN POST TRAUMÁTICA DE EXTREMIDADES*
}

\author{
Drs. Günther Mangelsdorff G. ${ }^{1}$, Cristián Taladriz R. ${ }^{1}$, Nicolás Pereira C. ${ }^{1}$, \\ Tomás Echeverría U. ${ }^{1}$, Carlos Domínguez C. ${ }^{2}$, Ricardo Roa G. ${ }^{1}$ \\ 1 Departamento de Cirugía Plástica y Quemados. Hospital del Trabajador. Asociación Chilena de Seguridad. \\ 2 Servicio de Urgencia. Hospital del Trabajador. \\ Santiago, Chile.
}

\begin{abstract}
Propeller flap in post-traumatic lower limb defects

Aim: To describe indications and results obtained in distal lower leg post traumatic reconstruction using pediculated perforator propeller flap. Patients and Methods: Prospective series of consecutive patients treated with propeller flaps between March 2011 and March 2014. Patient's characteristics, defects and flaps characteristics and post operative complications were recorded. Descriptive statistical methods were used in this study. Results: Eight patients were included with a median age of 40 years. The injuries were caused by degloving (one case) and fractures (seven cases), including four calcaneal fractures, two open tibial fractures and one ankle fracture. Three cases were reconstructed with posterior tibial artery pedicled perforator flaps, and five cases with peroneal artery perforator flaps. The median average area of the flaps was $55.8 \mathrm{~cm}^{2}$ (range $40-117.8 \mathrm{~cm}^{2}$ ). Coverage was achieved in all cases. No total flap necrosis was reported. Discussion: Propeller flaps are a useful tool in reconstruction of medium size defects of the distal third of the lower limb. They allow primary closure of the donor site and have a low rate of complications.
\end{abstract}

Key words: Lower leg, propeller flap, pediculated flap.

\section{Resumen}

Objetivos: Evaluar los resultados obtenidos en cobertura cutánea post traumática con colgajos pediculados basados en perforantes distales en extremidades inferiores. Pacientes y Método: Se realizó un registro prospectivo de todos los pacientes con lesiones post traumáticas tratados con colgajos perforantes pediculados en hélice entre marzo de 2011 y marzo de 2014. Se registró datos demográficos, características del defecto cutáneo, características de los colgajos utilizados, así como la presencia de complicaciones post operatorias. Se utilizó medidas de tendencia central y de dispersión para describir la serie de pacientes. Resultados: La serie analizada incluyó ocho pacientes con una mediana de 40 años (27-59 años). El mecanismo de lesión fue

\footnotetext{
Los autores no refieren conflictos de interés.

Correspondencia: Dr. Günther Mangelsdorff G. gmangelsdorff@hts.cl
}

*Recibido el 20 de mayo de 2014 y aceptado para publicación el 6 de octubre de 2014. 
desforramiento (un caso) y fracturas de la extremidad inferior (siete casos), de las cuales, 4 fueron fracturas de calcáneo, 2 fracturas expuestas de tibia y 1 fractura de tobillo. En tres casos se realizaron colgajos basados en perforantes de arteria tibial posterior y en cinco casos se utilizó perforantes de arteria peronea. El área de los colgajos tuvo una mediana de 55,8 $\mathrm{cm}^{2}$ (rango $40-117,8 \mathrm{~cm}^{2}$ ). Un caso presentó una necrosis distal, dos dehiscencia del borde distal y un hematoma. Se logró cobertura en todos los casos y la zona dadora fue cerrada en forma primaria. No hubo necrosis totales de colgajos. Discusión: El colgajo perforante pediculado de tipo propeller es una herramienta útil en la cobertura de defectos de extensión menor del tercio distal de la extremidad inferior con una baja tasa de complicaciones.

Palabras clave: Extremidad inferior, colgajo de perforante, colgajo pediculado, lesión de extremidades.

\section{Introducción}

La reconstrucción de defectos cutáneos distales en trauma de extremidades inferiores representa un desafío permanente, tanto por el extenso daño local secundario al trauma de alta energía, como por la escasa disponibilidad de tejidos locales. Para la reconstrucción en casos con exposición de material de osteosíntesis o de estructuras como hueso, tendón, vasos o nervios, se requiere aporte de tejidos mediante colgajos pediculados locales, regionales o colgajos libres mediante técnicas microquirúrgicas. $\mathrm{Si}$ bien los colgajos libres permiten resolver casos de lesiones extensas con seguridad y versatilidad, su complejidad habitualmente no se ve justificada en casos de lesiones más pequeñas ${ }^{1}$. Al planificar la reconstrucción en defectos pequeños o medianos, en la región distal de la pierna, tobillo y pie, se pueden considerar alternativas de colgajos locales, teniendo como principal limitación el daño local por el trauma. Las alternativas regionales, como el colgajo sural, permite el aporte de tejido más distante, probablemente menos traumatizado, pero con extensas cicatrices en zona dadora, con frecuencia requiriendo injertos, y además dejando como secuela anestesia del territorio del nervio sural ${ }^{2}$.

El desarrollo de los colgajos basados en perforantes ha permitido la movilización de segmentos cutáneos de tamaño variable basados en una o más perforantes, tanto en forma pediculada como libre ${ }^{3}$. Este tipo de colgajos se caracteriza por preservar la estructura y función muscular, por tener una vascularización confiable y por disminuir la morbilidad de zonas dadoras. En este grupo se encuentran los colgajos perforantes pediculados que se movilizan mediante la rotación en torno al eje dado por los vasos perforantes, logrando tanto la reparación del defecto cutáneo con piel distante a la lesión, como el cierre primario de la zona dadora habitualmente sin necesidad de injertos. El consenso de Tokio de 2011 define tres categorías para este tipo de colgajos: 1) Colgajo en hélice con pedículo subcutáneo: el pedículo es aleatorio, no se esqueletiza y la rotación de la isla de piel es de hasta $90^{\circ}$; 2) Colgajo en hélice con pedículo basado en perforante: el pedículo se basa en vasos perforantes, los cuales se esqueletizan logrando así una rotación de hasta $180^{\circ}$; 3) Colgajo en hélice supercargado: posee una perforante esqueletizada con rotación de la isla de piel hasta en $180^{\circ}$ y tiene además una arteria y vena adicionales en el otro extremo del colgajo, que luego son anastomosadas a vasos del lecho receptor una vez determinada la posición final del colgajo. Este puede ser el diseño original del colgajo, así como también puede ser utilizado como una medida de rescate frente a un colgajo con congestión venosa o isquemia ${ }^{4,5}$. En extremidad inferior distal estos colgajos se pueden basar en vasos perforantes de arteria peronea o tibial posterior, siendo habitualmente muy útiles los colgajos tipo 2 según consenso de Tokio, tanto por su irrigación como por su arco de rotación ${ }^{5}$.

A continuación presentamos una serie prospectiva de pacientes sometidos a colgajo de perforantes pediculados en hélice por déficit de cobertura en trauma de extremidades inferiores. El objetivo de este trabajo es evaluar los resultados del uso de colgajo de perforante de tipo propeller en lesiones traumáticas de extremidad inferior, así como describir sus indicaciones y aplicaciones.

\section{Pacientes y Métodos}

Se realizó un registro prospectivo de todos los pacientes con lesiones traumáticas distales de extremidad inferior que fueron sometidos a reconstrucción con colgajo perforante en hélice según protocolo abajo detallado, entre marzo de 2011 y marzo de 2014 (nivel de evidencia tipo IV). Se registró edad, sexo, mecanismo de lesión, tamaño del defecto, exposición de estructuras (ósea, material de osteosíntesis, tendón). Se evaluó las características de los colgajos utilizados (dimensiones y pedículo). El área del colgajo y del defecto se calculó de acuerdo a la fórmula de cálculo del área de la elipse $\left(\pi \times \mathrm{R}^{1}\right.$ $x \mathrm{R}^{2}$ ). Finalmente se procedió a registrar la presencia de complicaciones post operatorias y cirugías complementarias requeridas. Se utilizó medidas de tendencia central y de dispersión para describir la serie de pacientes. 
Nuestro protocolo de tratamiento en estos casos incluyó marcación preoperatoria con ecografía doppler color por radiólogo, marcación por cirujano con doppler y mapeo de perforantes en territorio tibial posterior o peroneo según el caso. Se diseñó el colgajo teniendo como referencia el tamaño del defecto y la ubicación de la perforante, considerando un ancho igual al defecto, y un límite proximal marcando desde la perforante una distancia dos centímetros mayor a la distancia entre el vaso seleccionado y el margen distal del defecto ${ }^{2}$. Bajo anestesia regional y sin isquemia, se realizó una incisión longitudinal en uno de los bordes del colgajo, con disección subfascial se identificó y disecó una o más perforantes hasta su emergencia del eje vascular, confirmando en esta etapa la ubicación del vaso seleccionado, el cual debe tener un diámetro de al menos $2 \mathrm{~mm}$, pulso palpable y doppler intraoperatorio audible. A continuación se completó la elevación de la isla de piel y rotación del colgajo en $180^{\circ}$ cubriendo el defecto y cerrando en forma primaria la zona dadora. Antes del cierre se chequeó el llene capilar del colgajo y doppler de la perforante. Se utilizó una curación no compresiva con adecuado posicionamiento post operatorio, considerando uso de órtesis inmovilizadora en casos de tobillo y pie, soporte elástico a las 3 semanas post operatorio y sistemas compresivos durante 6 meses.

Nuestra población de pacientes corresponde a trabajadores acogidos a ley de accidentes del trabajo, quienes están expuestos a compensación por las lesiones y secuelas.

\section{Resultados}

La serie analizada incluyó ocho pacientes, siete hombres y una mujer, con una mediana de edad de
40 años (rango 27-59 años). Las características de los pacientes se detallan en la Tabla 1 . Todos los pacientes sufrieron lesiones traumáticas, un desforramiento de la pierna y siete pacientes con fracturas de la extremidad inferior, de las cuales, cuatro fueron fracturas de calcáneo, dos fracturas expuestas de tibia y una fractura expuesta de tobillo. El área de los defectos tuvo una mediana de $13,4 \mathrm{~cm}^{2}$ (rango $4,7-28,3 \mathrm{~cm}^{2}$ ). Todos los casos tuvieron exposición ósea, tendínea o de materiales de osteosíntesis. Los colgajos efectuados tuvieron una mediana de área de $55,8 \mathrm{~cm}^{2}\left(40-117,8 \mathrm{~cm}^{2}\right)$. En cinco casos se utilizó colgajo perforante de arteria peronea y en tres casos perforantes de arteria tibial posterior. Se presentan secuencias de imágenes de dos casos (Figuras 1 y 2).

En todos los casos se logró cobertura completa del defecto. Un paciente portador de una fractura expuesta de pierna con material de osteosíntesis y osteomielitis crónica en tratamiento presentó una necrosis parcial del colgajo de 1 × $2 \mathrm{~cm}$ que requirió de aseo y un colgajo bipediculado para cobertura complementaria, presentando nuevamente dehiscencia con cultivos positivos para Pseudomona aeruginosa multirresistente por lo que finalmente se realizó retiro del material de osteosintesis con mejoría de la infección local. Dos pacientes presentaron una dehiscencia parcial distal del colgajo que fue manejada con resutura, uno de estos pacientes requirió de un injerto dermoepidérmico complementario. Un paciente presentó un hematoma postoperatorio que requirió revisión en pabellón (Tabla 1).

El tiempo operatorio tuvo una mediana de $120 \mathrm{~min}(80-190)$. En un paciente de la serie portador de tabaquismo severo se realizó una cirugía en dos tiempos, con el objetivo de evaluar en forma cierta la vitalidad de los segmentos más distales del colgajo previo a su rotación, asegurando así la cobertura completa del defecto en el segundo tiempo.

Tabla 1. Características de los pacientes

\begin{tabular}{|ccccccccc|}
\hline Edad & Sexo & Ubicación & Defecto & Colgajo & Área & Pedículo & Complicación & Reoperación \\
\hline 44 & M & Pierna & $2 \times 3$ & $4 \times 8$ & 38 & Tibial post. & No & No \\
\hline 39 & M & Pierna & $3,5 \times 3,5$ & $4 \times 10$ & 31 & Tibial post. & Dehiscencia & Resutura \\
\hline 35 & M & Calcáneo & $3,5 \times 10$ & $5 \times 21$ & 83 & Peronea & Necrosis parcial & Colgajo local \\
\hline 27 & M & Calcáneo & $3 \times 6$ & $4 \times 13$ & 41 & Peronea & No & No \\
\hline 36 & M & Calcáneo & $4 \times 4$ & $4 \times 12$ & 33 & Peronea & No & No \\
\hline 49 & M & Calcáneo & $2 \times 7$ & $5 \times 25$ & 118 & Peronea & No & No \\
\hline 59 & F & Tobillo & $5 \times 6$ & $6 \times 15$ & 71 & Tibial post. & Dehiscencia & Injerto \\
\hline 41 & M & Tobillo & $5 \times 8$ & $5 \times 19$ & 75 & Peronea & Hematoma & Drenaje \\
\hline
\end{tabular}

M: Masculino. F: Femenino. Tibial post: tibial posterior. Las dimensiones del defecto y del colgajo se expresan en ancho por largo en $\mathrm{cm}$. El área del colgajo en $\mathrm{cm}^{2}$. 

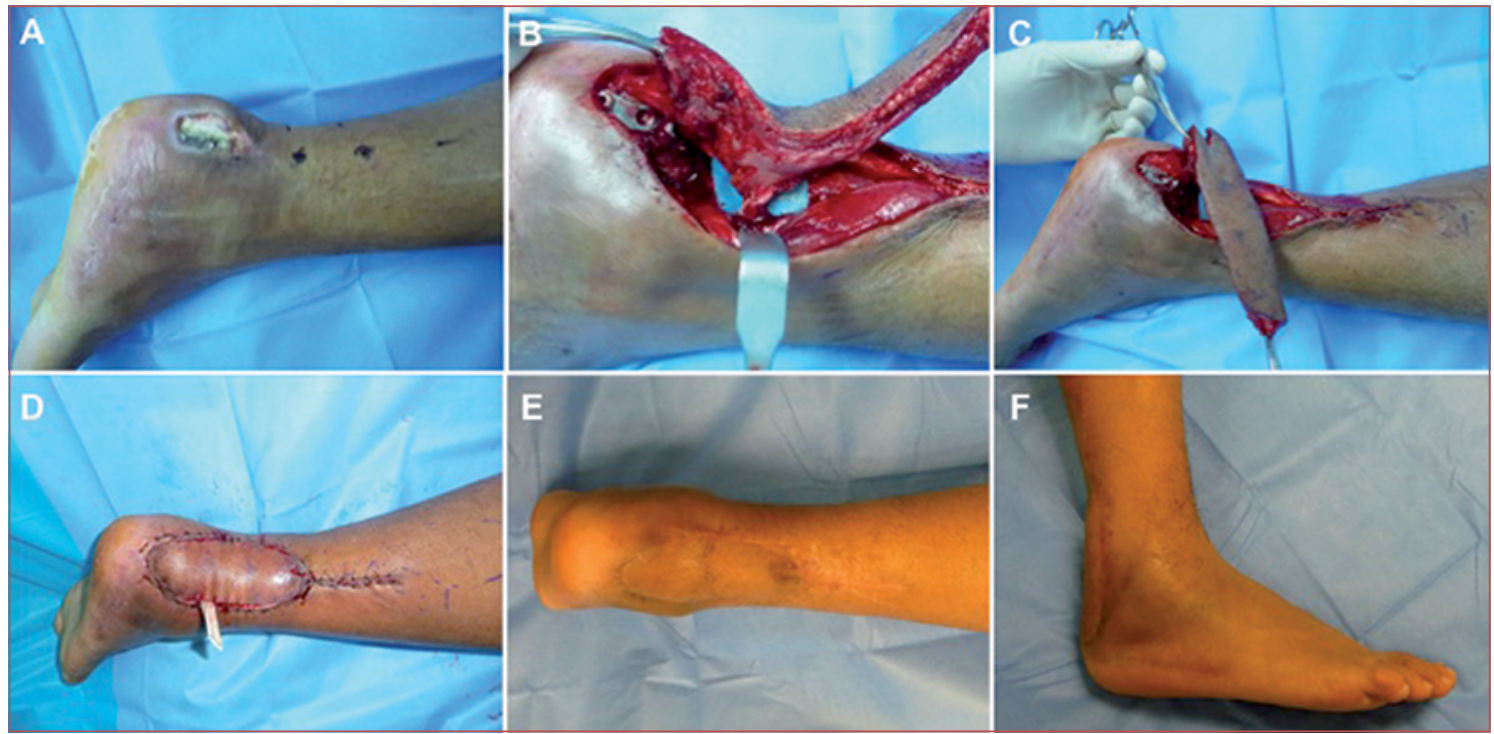

Figura 1. Paciente de 36 años con fractura de calcáneo que presentó necrosis cutánea y exposición de material de osteosíntesis. Se realizó cobertura con colgajo perforante pediculado en arteria peronea con evolución satisfactoria. Destacamos el contorno adecuado que se obtiene con este colgajo a nivel del tobillo. A) Defecto inicial y marcación de perforantes; B) Disección de perforante de arteria peronea y elevación del colgajo; C) Se muestra proceso de rotación del colgajo basado en perforantes; D) Cobertura completa del defecto con colgajo rotado en $180^{\circ}$; E) Resultado post operatorio alejado vista posterior; F) Resultado post operatorio alejado vista lateral, destaca adecuado contorno del tobillo.
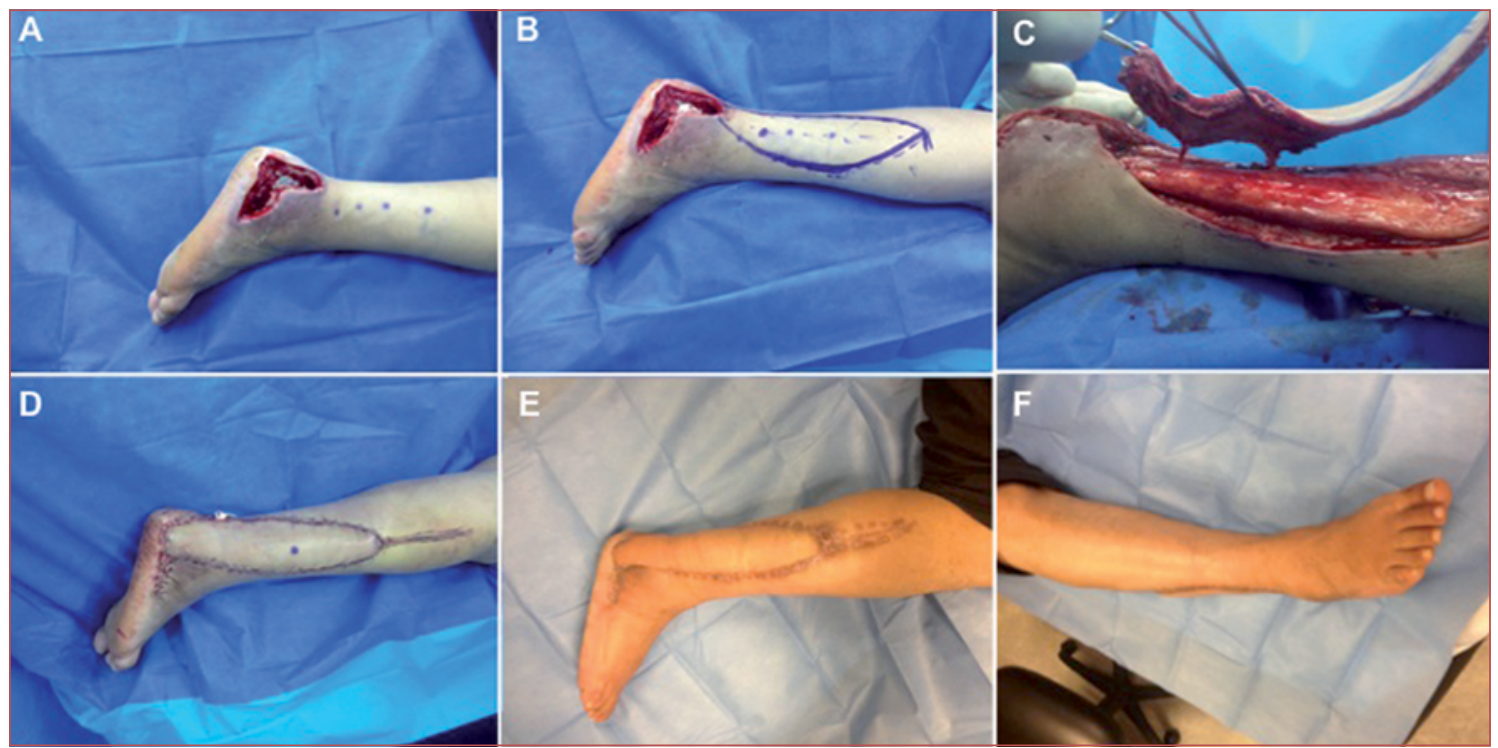

Figura 2. Paciente de 49 años, fractura de calcáneo que presentó necrosis cutánea extensa, exposición de placa de osteosíntesis e infección. Luego de aseos quirúrgicos se realizó cobertura con colgajo perforante pediculado en arteria peronea con evolución satisfactoria y buen contorno. A) Defecto resultante y marcación de perforantes; B) Marcación del colgajo; C) Disección de dos perforantes de arteria peronea, se escogió la perforante cefálica (a la derecha de la imagen) por tener mejor tamaño y pulso; D) Cobertura completa del defecto con colgajo rotado en $180^{\circ}$; E) Resultado post operatorio alejado vista lateral; F) Resultado post operatorio alejado vista anterior, destaca adecuado contorno del tobillo. 
La mediana de estadía hospitalaria fue de 29 días (rango 7-57 días). La mediana entre el momento del accidente y la cirugía fue 66 días (rango 1-162 días) y desde la cirugía reconstructiva al momento del alta hospitalaria fue de 22 días (rango 10-35 días). Se completó seguimiento en todos los pacientes con una mediana de 11 meses (rango 5-36). No se observó ulceración de los colgajos ni cicatrices inestables en el período de seguimiento.

\section{Discusión}

El déficit de cobertura de partes blandas secundario a trauma es una causa importante y frecuente de cirugía reconstructiva en las instituciones de salud ${ }^{4,6}$. Diferentes alternativas de cobertura de estos defectos pueden determinar un mejor pronóstico de las lesiones asociado a una menor morbi-morbilidad ${ }^{7}$. En extremidades inferiores, el tipo de colgajo a utilizar dependerá en gran medida de la localización del defecto (tercio proximal, medio o distal) y de su tamaño (grande, mediano o pequeño). De esta forma y tal como lo confirman múltiples autores, la primera elección para defectos de hasta $25 \mathrm{~cm}^{2}$ en el tercio proximal y tercio medio de la pierna son los colgajos cutáneos o fasciocutáneos locales random, y colgajos musculares de gastrocnemius medial y de músculo sóleo, que generalmente proporcionan una adecuada cobertura con baja morbilidad ${ }^{1}$. En cambio, en el tercio distal de la extremidad inferior las alternativas locales son reducidas. Los colgajos cutáneos random y colgajos fasciocutáneos de rotación son útiles en defectos pequeños, pero presentan rangos limitados de movilidad, por lo que en defectos de mayor tamaño habitualmente requieren uso de injertos en la zona dadora. En defectos por trauma, la disponibilidad del colgajo hemisóleo a pedículo distal habitualmente se ve reducida por la extensión del daño local. En el caso de defectos laterales del talón el colgajo axial calcáneo lateral resulta útil, pero a diferencia del colgajo presentado en este trabajo, el calcáneo lateral requiere injerto en zona dadora en nivel posterior del tobillo/talón lo que puede ocasionar coberturas inestables debido a la presión, fricción y cizalla que debe tolerar esa zona. Los colgajos pediculados basados en perforantes con diseño en hélice (propeller) son una buena alternativa en la cobertura de defectos del tercio distal, de tamaño mediano, asociado a bajas complicaciones de la zona dadora en circunstancias donde no es posible lograr una cobertura adecuada con colgajos locales ${ }^{1,8}$. En esta serie de pacientes observamos que los colgajos perforantes pediculados con diseño en hélice (propeller flaps) son útiles en la reconstrucción de defectos post traumáticos distales en extremidades inferiores, de tamaño mediano, en zonas con poca disponibilidad de tejidos locales que limitan las alternativas de reconstrucción, contrario a lo que ocurre en segmentos más proximales de la pierna. Entre sus principales ventajas debemos destacar que permiten movilizar segmentos de piel de características similares a la zona receptora, con adecuada irrigación y vitalidad, logrando utilizar piel más alejada del trauma, obteniendo así adecuada cobertura de defectos distales y cierre primario de zona dadora. Requiere planificación y diseño con ecografía doppler auxiliar, siendo muy importante una cuidadosa disección de los vasos perforantes. Siguiendo un protocolo como el que detallamos obtuvimos adecuada vitalidad de los colgajos, con un caso de necrosis parcial distal, siendo la principal complicación postoperatoria la dehiscencia distal de la herida. En estudios previos, es posible consignar tasas globales de complicación de un $42 \%$. La congestión venosa es la complicación más frecuente con un $17 \%$ de los casos aunque generalmente de resolución espontánea ${ }^{8}$.

Una alternativa de cobertura en casos similares a los presentados en esta serie es el colgajo sural a pedículo distal, ampliamente utilizado, descrito por Masquelet, una herramienta sin duda útil en cobertura distal de extremidades ${ }^{13,14}$. El colgajo sural a pedículo distal tiene reportada una mayor tasa de necrosis, la cual se ha descrito entre $59 \%$ y $39 \%$ para necrosis parcial y total aproximadamente ${ }^{5}$. Además sacrifica el nervio sural con la consecuente anestesia de la borde lateral del pie, lo cual es especialmente relevante en pacientes trabajadores activos que utilizan zapatos de seguridad, como aquellos que tratamos en nuestro centro. También se ha reportado el diseño de colgajo sural distal sin incluir el nervio sural ${ }^{13}$, aunque esto podría reducir el rango de cobertura al requerir punto pivote más cefálico, y su zona dadora es más extensa que el perforante de arteria peronea, con mayores requerimientos de injertos según el tamaño requerido. En nuestra serie no presentamos cobertura de talón, escenario donde el colgajo sural puede ser una mejor opción. En comparación al colgajo sural, en nuestra opinión el colgajo perforante de arteria peronea o tibial posterior presenta ciertas ventajas: su zona dadora es más reducida y habitualmente cierra en forma primaria, no requiere incluir el nervio sural, preservando así la sensibilidad protectiva de la cara lateral del pie, y tiene menor riesgos de necrosis. Al igual que lo publicado en otras series, no contamos con información que permita definir cuáles factores de riesgo pueden ser determinantes en la evolución de este tipo de colgajos, aunque sin duda la disección cuidadosa y adecuada del pedículo resulta esencial en la sobrevida del colgajo ${ }^{15,16}$. 
En nuestro centro utilizamos habitualmente colgajos libres en reconstrucción distal de extremidad inferior principalmente en defectos extensos, siendo las alternativas más utilizadas el colgajo perforante anterolateral de muslo, el colgajo muscular gracilis y el vasto lateral, estos últimos con uso de injerto dermoepidérmico. En defectos mayores debe considerarse además el latissimus dorsi. Probablemente, al igual que en los colgajos libres, la complicación más temida en los colgajos de perforantes pediculados en hélice (propeller) es la necrosis total del colgajo. Existen dos revisiones recientes, una de Gir et al. ${ }^{9}$, que evaluó 186 colgajos en 15 series de casos y una revisión de Nelson et a ${ }^{10}$, que incluyó 310 colgajos de 21 estudios. Ambas revisiones muestran tasas de necrosis parcial en el $11 \%$ de los colgajos con necrosis totales de $1 \%$ y $5,5 \%$ respectivamente, ${ }^{9,10}$. En nuestra serie no observamos casos de necrosis total. Dentro de las ventajas del colgajo de perforantes respecto a los colgajos libres destacamos que los primeros tienen un menor tiempo operatorio, no se requiere anastomosis microquirúrgica (aunque en ciertos casos puede adicionarse una anastomosis venosa para casos con congestión intraoperatoria) ${ }^{3}$, no dependen de la disponibilidad vasos receptores en la extremidad afectada y la zona dadora se encuentra adyacente al defecto sin necesidad de recurrir a otras localizaciones ${ }^{1}$.

En casos con factores de riesgo (ej. tabaquismo) puede considerarse la ejecución en dos tiempos para verificar la vitalidad de los segmentos más distales previo a su rotación ${ }^{8}$, aunque esto podría evitarse en caso de contar con tecnologías que permitan evaluación intraoperatoria de la irrigación, como por ejemplo la fluorescencia inducida por láser con verde de indocianina ${ }^{12}$.

Creemos importante considerar los colgajos pediculados basados en perforantes de arteria peronea y de tibial posterior, con diseño en hélice (propeller flap) para la cobertura en defectos cutáneos por trauma de extremidades inferiores, ya que son una buena alternativa al uso tanto de otros colgajos regionales como de colgajos libres para la cobertura de defectos distales de tamaño reducido en extremidades inferiores.

\section{Referencias}

1. Griffin J, Thornton J. Lower extremity reconstruction. SRPS Volume 11 Issue R1, 2009; pág 30-47.

2. Elsaftawy A, Jabłecki J, Domanasiewicz A, Paruzel M, Kaczmarzyk J, Kaczmarzyk L. Treatment possibilities of reverse-flow sural flap in covering the defects of lower extremities. Pol Przegl Chir. 2013;85;192-7.
3. Hallock GG. The propeller flap version of the adductor muscle perforator flap for coverage of ischial or trochanteric pressure sores. Ann Plast Surg. 2006;56:540-2.

4. Pignatti M, Ogawa R, Hallock GG, Mateev M, Georgescu AV, Balakrishnan G, et al. The "Tokyo" consensus on propeller flaps. Plast Reconstr Surg. 2011;127:716-22.

5. Mangelsdorff G, Rodríguez JR, Deichler MF, Hevia C, Hasbún A, Roa R. Colgajo perforante en hélice basado en arteria peronea para reconstrucción distal de extremidad inferior. Rev Chil Cir. 2013;65:433-7.

6. Masia J, Moscatiello F, Pons G, Fernández M, López S, Serret P. Our experience in lower limb reconstruction with perforator flaps. Ann Plast Surg. 2007;58:507-12.

7. Georgescu AV. Propeller perforator flaps in distal lower leg: evolution and clinical applications. Arch Plast Surg. 2012;39:94-105.

8. Schaverien MV, Hamilton SA, Fairburn N, Rao P, Quaba AA. Lower limb reconstruction using the islanded posterior tibial artery perforator flap. Plast Reconstr Surg. 2010;125:1735-43.

9. Gir P, Cheng A, Oni G, Mojallal A, Saint-Cyr M. Pedicled-perforator (propeller) flaps in lower extremity defects: a systematic review. J Reconstr Microsurg. 2012;28:595-602.

10. Nelson JA, Fischer JP, Brazio PS, Kovach SJ, Rosson GD, Rad AN. A review of propeller flaps for distal lower extremity soft tissue reconstruction: is flap loss too high? Microsurgery 2013;33:578-86.

11. Ramesha KT, Vijay J, Shankarappa M. Propeller flaps and its outcomes-a prospective study of 15 cases over two-years. J Clin Diagn Res. 2014;8:87-9.

12. Kanuri A, Liu AS, Guo L. Whom should we SPY? A cost analysis of laser-assisted indocyanine green angiography in prevention of mastectomy skin flap necrosis during prosthesis-based breast reconstruction. Plast Reconstr Surg. 2014;133:448e-54e.

13. Masquelet AC, Romana MC, Wolf G. Skin Island flaps supplied by the vascular axis of the sensitive superficial nerves: anatomic study and clinical experience in the leg. Plast Reconstr Surg. 1992;89:1115-21.

14. Baumeister SP, Spierer R, Erdmann D. A realistic complication analysis of 70 sural artery flaps in a multimorbid patient group. Plast Reconstr Surg. 2003;112:129140.

15. Jakubietz RG, Jakubietz MG, Gruenert JG, Kloss DF. The 180-degree perforator-based propeller flap for soft tissue coverage of the distal, lower extremity: A new method to achieve reliable coverage of the distal lower extremity with a local, fasciocutaneous perforator flap. Ann Plast Surg. 2007;59:667-71.

16. Innocenti M, Menichini G, Baldrighi C, Delcroix L, Vignini L, Tos P. Are There Risk Factors for Complications of Perforator-based Propeller Flaps for Lowerextremity Reconstruction? Clin Orthop Relat Res. 2014 Apr 5. [Epub ahead of print]. 